\title{
ECKART MENSCHING (1936-2007) E GLI STUDIOSI SUI QUALI SCRIVE
}

\begin{abstract}
Riassunto. - L'articolo è dedicato a Eckart Mensching (1936-2007), professore di filologia classica all'università di Berlino che ha dedicato, nelle riviste „Latein und Griechisch in Berlin“ e „Nugae“ da lui dirette, molti profili di studiosi della Berlino del 20esimo secolo. Qui si propone quello di tre famosi professori ebrei del 20esimo secolo che hanno dovuto lasciare la loro città e università, affrontando l'esilio con grande dignità: Eduard Norden, Felix Jacoby e Friedrich Solmsen.
\end{abstract}

Prima che sia troppo tardi, vorrei lasciare a questa rivista un ricordo di E. Mensching, deceduto il 21 luglio del 2007 e nato il 1 dicembre del 1936, figlio di un pastore evangelico e noto latinista e grecista, al quale la filologia classica internazionale e nazionale deve moltissimo, coltissimo e capace di giudizi profondi ed equilibrati.

Dal curriculum di E. Mensching emerge che la sua formazione classica è avvenuta in Isvizzera (Berna e Basilea) con una dissertazione su Favorino di Arelate (filosofo del secondo secolo d. C.) nel 1961 e che il suo rientro in Germania si ricollega con la libera docenza a Gottinga nel 1968, dopo la quale, nel 1970, ottenne la cattedra di filologia classica alla Technische Universität Berlin, dove svolse la sua molteplice attività quasi fino alla morte. Collaborava con molte istituzioni di didattica greca e latina, sia scolastiche, sia universitarie, era sensibilissimo a problemi linguistici, storici e di formazione.

Era redattore capo della rivista „Latein und Griechisch in Berlin“, un giornale di comunicazione e di informazione del „Deutscher Altphilologenverband", edito dalla illustre casa editrice Diesterweg di Francoforte. Ad essa si aggiunsero presto le „Nugae“ (,futilità“) zur Philologie-Geschichte, note di ulteriore approfondimento di temi trattati. E. Mensching chiude ogni volume con un indice di nomi, cenni di cronologia, indici del contenuto e anche di cenni ad altri volumi.

„Latein und Griechisch in Berlin“ informava puntualmente su novità e stimoli interessanti in campo del mondo classico, e non solo a Berlino: congressi di ogni genere, mostre, musei e scavi in tutto il mondo, libri recenti sul mercato, compresi fumetti e riviste nuove; in- 
formava anche su personalità emergenti, su compleanni di personaggi illustri, vivi e defunti, forniva ricette di cucina romana accanto a particolarità del latino parlato. A volte Mensching si trova anche a correggere false informazioni su progetti scientifici, su amicizie o su rapporti tra studiosi, ecc.

Le Nugae contengono piuttosto una panoramica esauriente e puntuale su studiosi e studi a livello internazionale e nazionale, biografie, corrispondenze e ricordi presentati in varie occasioni, ad es. anniversari, o estesi studi sulla didattica, su opere enciclopediche ecc. Ogni volumetto porta una dedica, di solito a un membro della famiglia Mensching e una volta a Solmsen (Nugae II, 1989) e un altra volta a una figlia di Norden (Nugae VI, 1993), e si chiude con un indice di nomi e altri elenchi.

Uno degli aspetti per noi più importanti di questi volumi è che spesso sono dedicati o contengono notizie relative a vittime del periodo nazista, agli studiosi costretti a emigrare (con le famiglie), a cambiare radicalmente vita, di solito perché ebrei. A loro Mensching tributa un grande riconoscimento. E solo un cittadino di Berlino che aveva accesso ai grandi archivi e alle biblioteche, poteva intraprendere queste ricerche che comprendevano anche notizie (con fotografie) sulle abitazioni degli studiosi, sugli edifici pubblici legati all'università e sulla stessa università. L'autore sapeva come muoversi per trovare notizie riguardanti progetti scientifici, cambiamenti all'interno del personale, chiamate, ma anche famiglie e amicizie dei docenti, ecc.

Qui vorrei ricordare in particolare i contributi di E. Mensching sugli studiosi Eduard Norden (1868-1941), Felix Jacoby (1876-1959) e Friedrich Solmsen (1904-1989) - quest' ultimo anche perché ho avuto la fortuna di conoscerlo personalmente e abbastanza bene, quando, durante i miei anni universitari a Francoforte egli, usufruendo una borsa Fulbright (1958), tenne un bellissimo seminario su Esiodo.

Lo studioso più illustre di quegli anni, costretto a una emigrazione forzata, perché capì abbastanza tardi la pericolosità del regime e il suo rischio personale, è Eduard Norden, generalmente considerato il migliore latinista del suo tempo, al quale Mensching ha dedicato giustamente molto spazio. ${ }^{1}$

\footnotetext{
${ }^{1}$ Nugae V, 1992, pp. $66 \mathrm{sgg}$. Ricordo brevemente le opere più importanti di Norden che tuttora sono presenti nelle biblioteche e sono state ristampate varie volte:

1898 Antkie Kunstprosa che riguarda il periodo dal $6^{\circ}$ sec. a. C. fino al rinascimento (3 volumi); 1903 P. Vergilius Naso: commento al libro VI dell' Eneide; 1910 Letteratura romana: Die lateinische Literatur, ihr Uebergang vom Altertum zum Mittelalter; 1913 Agnostos Theos. Untersuchungen zur Formgeschichte religiöser Rede; 1913 Josephus und Tacitus über Jesus Christus und eine messianische Prophetie (Kl. Schr. pp. 241-75); 1915 Ennius und Vergilius. Kriegsbilder aus Roms grosser Zeit; 1920 Die germanische Urgeschichte in Tacitus' Germania; 1924 Zur IV. Ekloge: Die Geburt des Kindes: Geschichte einer religiösen Idee; 1934 Alt-Germanien; 1939 Aus altrömischen Priesterbüchern.
} 
Norden ha passato gran parte della sua carriera a Berlino. Due volumi delle Nugae riguardano solo lui, il V (1992) e il VI (1993). Quest' ultimo („Erinnerungen an E. Norden und andere Beiträge“) contiene soprattutto ricordi di lui, in particlare quelli della moglie Marie che esaudì il desiderio (v. sopra) tra la fine del 1942 e l'inizio del $1944 .^{2}$ Mensching arricchisce questi testi con molte note e con un contributo personale. Di lui sono anche i „Nachträge“ (p. 153 sgg. ), dove si apprende ad es. che Norden suonava il pianoforte, che faceva volentieri passeggiate, che amava l'isola di Norderney, e si apprende anche (p. 157) che cercava di aiutare molte persone in difficoltà (v. sotto). „Nugae“ V sono dedicate interamente a Norden e alla sua opera in occasione dei 50 anni dalla sua morte.

Norden era nato in Frisia, aveva studiato Latino e Greco a Bonn e Berlino, laureandosi a Bonn (con F. Bücheler e H. Usener con una dissertazione su Varrone 1891) ${ }^{3}$. A diciotto anni ha ricevuto il battesimo nella chiesa evangelica. Nel 1885 divenne professore a Greifswald, dove sposò Marie Schultze, figlia del sindaco, nel 1899 fu chiamato a Breslau e nel 1906 alla Friedrich-Wilhelm-Universität di Berlino, dove rimase attivo fino al 1935 (v. sotto). Il culmine della sua carriera era il rettorato berlinese (1927/8), durante il quale fu anche festeggiato il suo 60 esimo compleanno. ${ }^{4}$ Data la sua elevata posizione all'università, ancora fino al 1933 la sua vita accademica scorreva abbastanza indisturbata, mentre già all'inizio di quell'anno ad alcuni colleghi fu proibito l'insegnamento (v. sotto). Norden continuò fino al suo pensionamento nel $1935^{5}$ e fu colpito dalle leggi razziali del 22 novembre 1938 , per cui dovette lasciare accademia e università e non potè più accedere alle biblioteche (anche se almeno cinque allievi continuavano a frequentarlo e a procurargli materiale per le ricerche, certo, a proprio rischio). Molto istruttive e di grandissimo interesse le lucide considerazioni di F. Solmsen sulla situazione politica del 1933 e sul tenace attaccamento di Norden alla patria e alla nazione. Per i ,altrömische Priesterbücher" (1936/7) non riuscì più a trovare un editore tedesco - furono stampati in Svezia. ${ }^{6}$ Forse capì solo allora la natura del regime, anche se già in passato si era adoperato per gli amici ebrei (p. 111), in particolare per Eduard Fraenkel, Georg Rohde e Friedrich Walter Lenz, ${ }^{7}$ cercando di trovare loro una sistemazione all'estero.

Nel 1939 Norden ottenne con grande difficoltà e grazie all'aiuto del fratello il permesso di emigrare in Svizzera, dopo aver venduto la casa e la biblioteca per finanziare questo esodo. A Zurigo morì il 13 luglio del 1941. Ovviamente adeguati necrologi potevano venire solo

${ }^{2}$ Latein und Griechisch 36, 1992, pp. 122 sg., Nugae VI pp. 8 sgg.

${ }^{3}$ In Varronis saturas Menippeas observationes selectae. Cfr. Latein und Griechisch 36, 1992, p. 206.

${ }^{4}$ Nugae V, pp. 89 sgg.

${ }^{5}$ Nugae V, p. 108.

${ }^{6}$ Nugae V, p. 158 sg., v. sotto.

${ }^{7}$ Nugae V, p. 111 
dall'estero: ${ }^{8}$ ricordo quelli di E. Howald sulla „Neue Züricher Zeitung" e di H. Fuchs sulle „Basler Nachrichten“. A questi si aggiunsero tre necrologi anonimi dal mondo anglosassone: uno su "The Times“, probabilmente di Eduard Fraenkel e due dagli Stati Uniti, in cui Mensching individua gli autori George Lincoln Hendrickson e, con un punto interrogativo, Ernst Riess; ${ }^{9}$ infine uno da un ,insider' sul piccolo giornale locale ,Rheiderland', probabilmente scritto ancora durante l'èra nazista. ${ }^{10}$

Informazioni, giudizi politici ad altri, inquadramenti storici ecc. di Mensching sono preziosissimi: per quanto riguarda Norden, egli fornisce osservazioni su tutte le edizioni di ogni singola opera, giudizi di vari colleghi ed amici (ai quali Norden chiese spesso una lettura e un giudizio, prima di mandare $\mathrm{i}$ testi in tipografia ${ }^{11}$; così veniamo a sapere di recensioni, anche su quotidiani e di ristampe (di solito con aggiunte); siamo informati sui rapporti con gli amici stretti come C. Cichorius, R. Heinze e soprattutto F. Jacoby (v. sotto), e sulle relazioni amichevoli con U. von Wilamowitz (morto nel 1931), Ed. Meyer (morto nel 1930), Werner Jaeger (morto nel 1961) e Franz Skutsch, dalla cui prematura morte (1912) Norden rimase molto colpito. Mensching parla dei riconoscimenti accademici, come le lauree honoris causa: tra quelle che lo emozonarono di più, quella della facoltà di teologia di Bonn nel $1919^{12}$ (Nugae V., p. 68) e quella di Cambridge il 4 giugno $1929 .{ }^{13}$

Norden apparteneva a diverse Accademie (ad es. quella prussiana dal 1912, quella del Thesaurus, dal 1923), di molte era membro corrispondente (ad es. di Vienna, Leningrado, Lund, Uppsala e Göteborg, di Krakovia, Milano e Napoli), fece vari viaggi, nella stessa Germania e all'estero, ad es. in Italia, ma anche negli Stati Uniti.

Interessante è il suo stretto rapporto con la casa editrice Teubner e anche con la sua tipografia. Stampare e ristampare un'opera di Norden non dev'essere stato cosa facile: consegnava di solito pezzi di manoscritto, al volume in corso di stampa, forniva ripensamenti, correzioni ed aggiunte („Berichtigungen“, „Nachträge“) ecc. (Nugae V, p. 70 sgg. )

Naturalmente, Mensching fornisce molte informazioni sull'insegnamento di Norden: pare che non abbia mai perso di vista il panorama della scuola secondaria (Nugae V, p. 156); il suo insegnamento era sempre rivolto in prima linea a futuri insegnanti di liceo, e negli esami apprezzava soprattutto la capacità di fornire risposte adeguate.

${ }^{8}$ Tutte queste notizie in Nugae V, p. 105 sgg. e in Latein und Griechisch 36, 1992, pp. $224 \mathrm{sgg}$.

${ }^{9}$ Latein und Griechisch 36, 1992, p. 225 sgg.

${ }^{10}$ Latein und Griechisch 36, 1992, pp. 229 sgg.

${ }^{11}$ Nugae V, p.115 sg.

${ }^{12}$ Nugae V, p.68

${ }^{13}$ Latein und Griechisch 36, 1992, p. 205 
Un certo interesse suscitano discorsi di Norden riportati da Mensching, in particolare quelli pronunciati durante il periodo del suo rettorato, tra cui uno intitolato „Heldendichtungen“ (Nugae V, p. 94 sgg.) di tono patriottico, con l'interpretazione del ciceroniano se offerre ad mortem, con il riferimento a Socrate, a Cristo e al sacrificio dei giovani soldati per la patria. Dalle pagine di Mensching acquistano risalto anche i nuovi compiti, di carattere amministrativo e pratico, viaggi di servizio e di rappresentanza e nuovi aspetti umani.

$* * * *$

Felix Jacoby presenta un quadro che sotto molti aspetti assomiglia a quello di Norden. Era di Magdeburg, auche lui battezzato con rito evangelico, ha frequentato un Pädagogicum cattolico. Era un allievo, a Berlino nel 1901, di Hermann Diels e Ulrich von Wilamowitz. Al periodo successivo sono da attribuire diverse voci della Pauly Wissowa. ${ }^{14}$ Ottenuto la libera docenza a Breslau, fu chiamato a Kiel nel 1906/7 come ordinario. Ha partecipato alla Prima Guerra Mondiale e ottenuto 1'"Ehrenkreuz für Frontkämpfer".

Come Norden era troppo conservatore per riconoscere subito la pericolosità del regime nazista. Dopo il pensionamento forzato (v. sotto) Jacoby si ritirò a Finkenkrug (Berlino), e qui - in sua assenza - l'interno della sua casa fu barbaramente demolito nella „Kristallnacht“ del novembre $1938,{ }^{15}$ anche se gli uomini della SA non riuscirono a scassinare la cassaforte che conteneva l'intero schedario dei Fragmenta degli storici greci e manoscritti. Davanti alla sua casa distrutta, Jacoby decise di trasferirsi con la moglie al Christ Church College di Oxford, aiutato, anche finanziariamente, soprattutto da Eduard Fraenkel, ma trovando, come Norden, grossi ostacoli all' emigrazione e naturalmente perdendo qualsiasi pagamento dalla Germania; ${ }^{16}$ il trasferimento divorò quasi l' intero patrimonio degli Jacoby che tornarono a Finkenkrug nel 1956, dove Felix morì il 21 novembre del 1959.

Mensching riporta nelle Nugae tre scambi di lettere tra Jacoby e varie autorità, lettere che rivelano il carattere deciso dello studioso, corretto e non disposto a compromessi. La corrispondenza tra Jacoby e il ministero prussiano ${ }^{17}$ circa problemi di pensionamento, prepensionamento e alla fine pensionamento forzato con obbligo di rinuncia all'insegnamento, ha inizio nel 1933 e si chiude nel 1934, seguita dal colloquio con un referente ministeriale nell'aprile dello stesso anno e il successivo congedo di Jacoby nell'aprile del 1935. Mensching riferisce inoltre di una grave diffamazione dello studioso pubblicata sulla

14 ad es. Herodot nei Supplementa II, 1913, coll. 205-520, Hieronymus von Kardia, Halb. 17, 1913; Ktesias, Halb. 22, 1922.

${ }^{15}$ Nugae V, p. 45.

${ }^{16}$ Solo dal 1 aprile del 1950 Jacoby ricevette di nuovo la sua pensione (Nugae II, p. $53)$.

${ }^{17}$ Nugae V pp. 49 sgg. 
„Philologische Wochenschrift“ del 1935 che gettò Jacoby in una profonda depressione, dalla quale però, al contrario di Norden, riuscì a riprendersi. L'accusa riguardava l'edizione dellaTeogonia di Esiodo definita nella recensione semplicemente „undeutsch“" ${ }^{18}$.

Interessante è poi la corrispondenza riportata da Mensching, con il presidente dell'Accademia prussiana, M. Planck, che il primo dicembre 1938 chiese a tutti i membri di origine ebraica di dare le dimissioni. Jacoby rispose con una lettera ufficiale a dimissioni avvenute e Planck replicò con dignità ${ }^{19}$ (Nugae II, p. 41), esprimendo oltre al dispiacere la sua gratitudine e la sua stima. L'Accademia di Gottinga chiese invece a Jacoby di rassegnare le sue dimissioni su un formulario prestampato. Entrambe le Accademie gli offrirono di rientrare dopo il suo ritorno in Germania.

Mensching riporta infine la corrispondenza nel maggio 1938 con l'allora direttore della casa editrice Weidmann, Dr. U. Hillmann, sull'edizione dei „Frammenti degli storici greci“ inseparabilmente legata al nome di Jacoby. Weidmann non aveva intenzione di continuarla con un autore ebreo. La risposta di Jacoby (in Nugae IV) contiene sottolineature e commenti al margine, e porta alla rottura drastica del rapporto editoriale.

Com'è noto, questa grande e ammirevole edizione ha come fondamento la raccolta di frammenti della Cronaca del grammatico Apollodoro di Atene (FrGrHist II B Nr. 244, v. Nugae II, p. 28 sgg. ), che consiste in una cronologia degli avvenimenti più importanti della storia greca, e poi il „Marmor Parium“, una cronaca di età ellenistica incisa su marmo di cui conserviamo due pezzi, trovati nel 1627 a Smirna (FrGrHist II B Nr. 239). Della preziosa edizione dei frammenti si incaricò E. J. Brill di Leiden ${ }^{20}$ e fu stampato quasi interamente in tempi della guerra (cfr. la nuova premessa in III A „Horographie und Ethnographie“", Oxford 1940). ${ }^{21}$

Vorrei chiudere questo breve ricordo con le parole dello stesso Mensching (Nugae V, p. 45): „Jacoby's Lebenswerk, das in englischer und deutscher Sprache, monumental' zu nennen naheliegt, verliert gewiss nicht an Eindrücklichkeit, wenn man ein wenig über die Umstände weiss, unter denen es entstanden ist".

$* * * *$

${ }^{18}$ Nugae II,p.28

${ }^{19}$ Nugae II, p.41

${ }^{20} \mathrm{La}$ stampa fu resa possibile anche grazie agli interventi di A. G. Roos (Groningen), E. R. Doods, H. Last, E. Fraenkel, G. Murray, H. T. Ward-Gerry (Oxford), M. Rostovtzeff (Yale) e Kenneth Sisams della Clarendon Press

${ }^{21}$ Molti anni dopo la morte di Jacoby nel 1991, il lavoro fu ripreso sotto la guida di G. Schepens e G. A. Lehmann, poi di H. J. Gehrke e F. Worthington. 


\section{Friedrich Solmsen}

Figlio del noto linguista Felix, Friedrich Solmsen studiò nella città natale Bonn, a Heidelberg e Berlino, dove conobbe non solo Wilamowitz, ${ }^{22}$ ma anche il suo futuro maestro Werner Jaeger, dal quale apprese l'interesse per la filologia e filosofia. Si laureò nel 1928 sotto la guida dei due studiosi con la dissertazione „Die aristotelische $\mathrm{Me}$ thodenlehre und die spätplatonische Akademie", pubblicata nella serie di „Neue Philologische Untersuchungen“ 4, rivista fondata da Jaeger. Nel 1929 conseguì la libera docenza con un lavoro sull'oratore Antifonte. ${ }^{23}$ A proposito della laurea e della libera docenza Mensching coglie l'occasione per rifierire ampiamente su questi lavori, sulla procedura di ottenere i due titoli, sulla vita accademica in Germania, le chiamate e la distribuzione delle cattedre a Berlino ed altrove, particolari qui forse meno rilevanti. E' invece ben rilevata l'immensa capacità lavorativa di Solmsen che fino al 1933 adempì in modo eccellente tutti gli obblighi ed oneri del giovane assistente, ma vide chiaramente il pericolo che avrebbe corso restando nella Germania nazista (v. sopra).

Nell'estate del 1930 gli fu affidato un seminario greco e da allora tenne ogni semestre un ciclo di lezioni e un seminario di argomento greco o latino fino alla sospensione dall'insegnamento nel 1933 e al divieto pressoché totale di pubblicare ${ }^{24}$ e di frequentare biblioteche.

Dopo essersi sposato con Lieselotte nel 1932, abbandonò nel 1933 Berlino, passò un periodo a Cambridge, poi si diresse negli Stati Uniti, lavorando prima all'Olivet College di Michigan (1937-40) come professore di filosofia, poi come filologo classico a Ithaka/New York (1940-62), a Madison/Wisconsin (1962-64) e infine a Chapel Hill/North Carolina (1964-74). Dovette ripercorrere di nuovo alcuni stadi già superati nella carriera tedesca, ${ }^{25}$ ma recuperò presto e continuò a lavorare su Platone ed Aristotele. ${ }^{26}$

Dopo la guerra tornò in Germania, ma non per sempre, bensì, come nel 1985 (v. sopra), per periodi più o meno lunghi (specialmente a Karlsruhe, dove abitavano parenti delle moglie), mentre pubblicava in due lingue su riviste tedesche e, a prescindere da diversi saggi, curava per Oxford l'edizione di Esiodo (1970, rist. 1983), poi il volume „Isis among the Greeks and Romans" (The Martin Classical Lectures, 1979) e alcuni saggi (dal 1975 in poi) nell'ambito della prima letteratura cristiana (Nugae III, 1990, n. 122).

${ }^{22}$ Più tardi pubblicò U. v. Wilamowitz, in His ten Years, Greek Rom. Byz. Stud. 20, 1979, pp. $89 \mathrm{sgg}$

${ }_{23}$ Antiphonstudien. Untersuchungen zur Entstehung der attischen Gerichtsrede, Neue Phil. Studien 8

${ }^{24}$ Eccezione fatta (grazie all'editore W. Kroll) per alcuni articoli della Pauly Wissowa.

25 il Ph. D. a Cambridge nel 1936

${ }^{26}$ 1942: Plato's Theology; 1960 Aristotele's System of the Physical World. 1962 Aisthesis in Aristoteles and Epicur; 1967 Electra and Orestes. V. anche sull'età dei sofisti: Intellectual Experiments of the Greek Enlightenment 1975. 
Gli fu conferita la laurea honoris causa dalle università di Kiel e di Bonn ed era membro di varie Accademie, quella dei Paesi Bassi, dell'American Philosophical Society dal 1966, dell' American Academy of Arts and Sciences dal 1968 e dell'Accademia britannica dal 1973, infine del Deutsches Archäologisches Institut.

Vorrei concludere queste considerazioni con le commoventi parole che posso solo sottoscrivere di Helen F. North in Ward W. Briggs, Biograph. Diet. of North Am. Classics 1994, pp. 604 sgg.: „As a teacher he was unfailingly patient, unhurried, generous with both criticism and encouragement".

Meritano un ricordo particolare le mogli di questi tre studiosi, Marie, Margarete e Liselotte, delle quali Mensching mette debitamente in rilievo l'importanza per aver condiviso la sorte dei mariti senza esitazione, con coraggio e dignità. E merita un grande elogio lo stesso Eckart Mensching che ha sempre profuso le sue grandi capacità al servizio di altri studiosi della sua disciplina e la diffusione di questa. 